\title{
Usefulness of Pharmacogenetic Analysis in Psychiatric Clinical Practice: A Case Report
}

\author{
Manuel A. Franco-Martin ${ }^{1,2}$, Francisco Sans ${ }^{1}$, Belen García-Berrocal ${ }^{2,3}$, Cristina Blanco ${ }^{1}$, Carlos Llanes-Alvarez ${ }^{1}$, \\ María Isidoro-García ${ }^{2,4}$ \\ ${ }^{1}$ Department of Psychiatry and Mental Health, Zamora Hospital, Zamora, ${ }^{2}$ Biosciences Institute of Salamanca and ${ }^{4}$ Department of Medicine, \\ University of Salamanca, ${ }^{3}$ Department of Clinical Biochemistry, University Hospital of Salamanca, Salamanca, Spain
}

\begin{abstract}
There are many factors involved in the effectiveness and efficiency of psychiatric drug treatment. One of them is psychotropic drug metabolism, which takes place mostly in the liver through the P450 enzyme system. However, there are genotypic variants of this system's enzymes that can directly affect both the efficacy and the onset of side effects of a given therapeutic regimen. These genotypic changes could partly explain the lack of efficacy of treatment in certain patients. We report the case of a patient diagnosed with bipolar type I disorder that presented multiple and frequent manic episodes in which the efficacy and tolerability of several pharmacological regimens with mood stabilizers and antipsychotics was scarce. The choice of medical treatment should be based on its efficacy and side effect profile. This decision can be made more accurately using the information provided by pharmacogenetic analysis. This case illustrates the importance of pharmacogenetic studies in clinical practice. The results of pharmacogenetic analysis helped to decide on a better treatment plan to achieve clinical improvement and reduce drug-induced adverse effects.
\end{abstract}

KEY WORDS: Bipolar disorder; Antipsychotic drugs; Pharmacogenetics; Cytochrome P450; Individualized medicine.

\section{INTRODUCTION}

Bipolar type I disorder is a frequently severe and long-term mental disorder, with a 12-month prevalence between $0.5 \%{ }^{1)}$ and $1.2 \% .^{2)}$ Psychopharmacologic treatment seeks to achieve effective control of affective or psychotic symptoms and mood stabilization, with the ultimate goal of normalizing the patient's life and minimizing the risks associated with the disease. The most frequently used drugs are mood stabilizers and antipsychotic drugs, alone or in combination. ${ }^{3)}$ However, clinical experience shows that there is wide interpatient variability in psychopharmacological treatment response, which could lead to an irregular clinical course and a poor prognosis. ${ }^{4,5)}$ This variability in response may be associated with diverse in-

Received: January 2, 2017 / Revised: February 13, 2017

Accepted: May 19, 2017

Address for correspondence: Manuel A. Franco-Martin, MD, $\mathrm{PhD}$

Department of Psychiatric and Mental Health, Zamora Hospital, Av. Hernán Cortés 40, Zamora 49021, Spain Tel: +34-980-520200, Fax: +34-980-517431

E-mail: mfm@intras.es, mfrancom@sanidadcastillayleon.es ORCID: https://orcid.org/0000-0002-3639-2523 dividual factors such as diet, polypharmacy, or genetic variations that could alter the activity of psychotropic-drug-metabolizing enzymes and protein transporters leading to individual differences in plasma concentrations of the active substance and, consequently, to such response variability. ${ }^{5}$ To deal with this, clinicians often need to switch from one therapeutic regimen to another based on lack of efficacy, low tolerance or non-adherence. ${ }^{3,6)}$ The switching process must consider confirmation of compliance, optimize the benefits of the drug being used before starting the replacement, ${ }^{7}$ and take into account the pharmacokinetic and pharmacodynamic characteristics of the drugs used. Accordingly, the US Food and Drug Administration (FDA) recommended that patients should be subjected to a genetic test prior to the administration of certain antipsychotic drugs such as aripiprazole or clozapine. ${ }^{7,8)} \mathrm{A}$ retrospective assessment of the value of pharmacogenetics for prescribing psychotropic drugs has shown that inappropriate medication selection has generated an increase in the use of healthcare resources associated with mental disorders. ${ }^{9}$

Pharmacogenetic studies provide information about

(ㄷ) This is an Open-Access article distributed under the terms of the Creative Commons Attribution Non-Commercial License (http://creativecommons.org/licenses/by-nc/4.0) which permits unrestricted non-commercial use, distribution, and reproduction in any medium, provided the original work is properly cited. 
the influence of genetic factors in drug transport and metabolism, which helps in the choice of an appropriate drug treatment that maximizes therapeutic efficacy while minimizing side effects. ${ }^{10)}$

Regarding psychotropic drugs, their metabolism is mainly hepatic, via cytochrome P450, so they may be affected by competitive interactions with other drugs that use the same metabolic pathway, either as substrates, inductors or inhibitors. ${ }^{11)}$

In the case of antipsychotics, cytochrome P450 enzymes CYP2D6, CYP1A2, and CYP3A4/5 are the most relevant, and polymorphisms of the alleles of these proteins are associated with altered plasma levels of medication. ${ }^{12)}$ Approximately $40 \%$ of antipsychotics are metabolized via CYP2D6, 23\% via CYP3A4 and $18 \%$ via CYP1A2. ${ }^{13)}$ Specifically, CYP2D6 is involved in the metabolism of haloperidol, risperidone, olanzapine, and aripiprazole; CYP1A2 is involved in the metabolism of clozapine and olanzapine; and CYP3A4 takes part in the metabolism of haloperidol, clozapine, quetiapine, ziprasidone and aripiprazole. ${ }^{14-16)}$ It is estimated that between $10 \%$ and $20 \%$ of the western population have genetic variants that affect the functioning of these enzymes. ${ }^{13)}$ Consequently, the standard dose of a psychoactive drug may result in plasma concentrations that may be sub-therapeutic or toxic depending on the patient's cytochrome P450 genotype. Hence, the results of pharmacogenetic studies could significantly contribute to appropriate selection of drug treatment. ${ }^{17,18)}$ In addition, many specific transport proteins are located in the BBB (blood-brain barrier) and intestine. MDR1 multidrug resistance 1, P-glycoprotein (P-gp) or ABCB1 is an ATP-binding cassette superfamily protein involved in pharmacokinetics of diverse antipsychotics. Genetic variants in MDR1 gene can affect the efflux of drugs out of the cell or the permeability of the BBB. So, Pharmacogenetic analysis of MDR1 can contribute to improve application of personalized medicine in schizophrenia treatment.

However, despite the FDA's recommendation on the use of pharmacogenetics and the results of clinical studies showing its usefulness to establish the most effective treatment, it is hardly used for assessing the hepatic metabolism of antipsychotics.

In this case report, the pharmacogenetic study of cytochrome P450 and the efflux transporter MDR1 provided valuable information. The metabolic capacity of the pa- tient have been of great importance to achieve the stabilization of a complex clinical situation that was causing great suffering to the individual, impoverishing his quality of life and involving high costs for the healthcare system.

\section{CASE}

We present the case of a 33-year-old Caucasian male patient. During periods of clinical stability, he used to work with high performance. He has no siblings, practically no social life and few leisure interests. He smokes 20 cigarettes a day and denies regular consumption of recreational drugs or alcohol, although he admits to the occasional use cannabis, which has triggered episodes of behavioural disorders. He has spent long periods off work due to his psychiatric condition, which has led to the loss of his current job.

He refers no psychiatric family history. Somatic history shows obesity and gynecomastia.

Past psychiatric history: he was first diagnosed with bipolar type I disorder in 2003, at the age of 20 years, and was admitted to a psychiatric unit for the first time in 2006. From the clinical perspective, he suffers a very unstable condition with a poor and slow response to treatment and frequent relapses involving startling manic symptoms and severe behavioural disorders. Besides, he has experienced severe drug-induced side effects, the most remarkable being extrapyramidal symptoms, sedation, weight gain and dyslipidaemia. Because of the numerous and troublesome adverse effects, the patient has become reluctant to take medication, which has led to irregular treatment adherence and frequent relapses. Altogether, he has been admitted 18 times since 2006, with an average of twice a year. Thirteen of these admissions were to the acute unit of the Department of Psychiatry in Zamora Hospital, making a total of 256 days. The remaining 5 admissions were to the convalescence unit of the same department, involving a total of 41 days. This is strong proof of the great complexity and difficulty in achieving clinical stabilization of the patient. Moreover, keeping the patient in the inpatient care unit became an almost constant need from the months of November 2014 to March 2015, due to the combination of poor clinical response and the numerous side effects of the medication. 
Table 1. Treatment plan in March 2015, prior to pharmacogenetic analysis

\begin{tabular}{|c|c|c|c|c|c|c|c|c|c|c|c|c|c|c|c|c|}
\hline \multirow{2}{*}{ Drug } & \multicolumn{16}{|c|}{ Cytochrome P450 } \\
\hline & $1 \mathrm{~A} 1$ & $1 \mathrm{~A} 2$ & 1B1 & $2 \mathrm{~A} 6$ & $2 \mathrm{~B} 6^{*}$ & $2 \mathrm{C} 8$ & $2 \mathrm{C} 9^{*}$ & $2 \mathrm{C} 19$ & 2D6 & $2 \mathrm{E} 1$ & $3 \mathrm{~A} 4$ & $3 \mathrm{~A} 5^{*}$ & $3 \mathrm{~A} 7$ & $4 \mathrm{~B} 1$ & No CYP & MDR1* \\
\hline Clonazepam & & & & & $\begin{array}{l}\text { Ind } \\
\text { Inh }\end{array}$ & & & & & & S & & & & & \\
\hline Lithium & & & & & & & & & & & & & & & S & \\
\hline Carbamazepine & & $\begin{array}{c}\text { S } \\
\text { Inb } \\
\text { Ind }\end{array}$ & & Ind & $\begin{array}{l}\text { S } \\
\text { Ind }\end{array}$ & $\begin{array}{c}\text { S } \\
\text { Ind }\end{array}$ & Ind & $\begin{array}{l}\text { Ind } \\
\text { Inh }\end{array}$ & & & $\begin{array}{c}\text { S } \\
\text { Ind }\end{array}$ & $\begin{array}{c}\text { S } \\
\text { Ind }\end{array}$ & S & & & \\
\hline Paliperidone & & & & & & & & & & & & & & & $x$ & $x$ \\
\hline Olanzapine & & $\mathrm{S}$ & & & & & Inh & Inh & $\begin{array}{c}\text { S } \\
\text { Inh }\end{array}$ & & Inh & & & & & $x$ \\
\hline
\end{tabular}

No CYP, no use of P450 pathway; Ind, inductor; Inh, inhibitor; S, substrate.

*Enzymes altered in this patient.

\section{Mental Status Examination (March 2015)}

The patient was conscious and well oriented, but showed partial insight. He suffered from reduced cognitive capacity, with cognitive slowing, inattention and distractibility. His mood oscillated from euphoria and grandiosity to irritability and dysphoria, which made his personal approach inappropriate. He exhibited pressure of speech, with racing thoughts and, occasionally, flight of ideas, which caused his communication to be incoherent. He felt restless, with episodes of psychomotor agitation and frequent verbal and physical aggressiveness, disruptive behaviour, and no sense of social distance. $\mathrm{He}$ had global impressions of severe insomnia, although he did not complain about it because he did not feel tired. He denied experiencing hallucinations or delusional thoughts, but revealed clear megalomaniac feelings and expressions.

\section{Investigations}

An electroencephalographic record conducted in March 2006 showed normal bioelectric brain activity. In May 2012, the results of a simple contrast computed tomography scan reported no abnormal findings.

\section{Pharmacological Treatment}

The poor and short lasting response to pharmacological treatment has led the patient to undergo different drug trials, combining mood stabilizers (lithium, valproic acid and carbamazepine), antipsychotics and benzodiazepines. Haloperidol and atypical antipsychotics such as risperidone, olanzapine, paliperidone, quetiapine, aripiprazole or asenapine have been tested, the latter specifi- cally indicated for the treatment of moderate to severe acute mania associated with bipolar disorder. Poor efficacy and tolerability (including weight gain, metabolic disorders, sexual dysfunction and persistent sedation) were present throughout most of the antipsychotic treatment. This situation became more apparent during the months of January, February and March 2015, when the patient was admitted on an almost continuous basis (Table 1).

One of the reasons for antipsychotic treatment failure is poor compliance secondary to tolerability issues. In fact, the patient's family suggested it as a possibility and, therefore, the first readmission was attributed to this problem. However, this probability was ruled out by supervising medication intake during the admissions.

Accordingly, a pharmacogenetic study was requested in March 2015, after performing a theoretical drug interaction analysis to assess the different pathways involved in the metabolism of the prescribed drugs and to identify the enzymes' corresponding drug substrate. The genes eligible for genotyping analysis were identified according to this complex theoretical analysis. In this case, we conducted a molecular analysis of gene variants CYP2B6 $\left({ }^{*} 6\right), \operatorname{CYP} 2 C 9(* 2, * 3), \operatorname{CYP} 2 C 19(* 2, * 3, * 17), C Y P 2 D 6(* 2$, $\left.* 3, * 4, * 5, * 6, * 7, * 8, * 9, * 10, * 12 *{ }^{*} 14, * 17, * 29, * 41, X \mathrm{~N}\right)$, CYP3A4 $\left({ }^{*} 1 B\right), C Y P 3 A 5\left({ }^{*}\right)$ and MDR1 (3435 C>T) (Table 2). The DNA sample was obtained from peripheral blood extraction and the analysis was performed using a Compaq MagnaPure system. CYP2D6 genotyping was performed using microarrays (Infinity, AutoGenomics, Palex, Spain) after PCR amplification. For other genes, a real-time PCR method was used, alongside fluorescence resonance en- 
Table 2. Pharmacogenetic analysis: genes tested, alleles identified and expected effect on protein function

\begin{tabular}{lccc}
\hline Gene & Alleles studied & Genotype & Expected phenotype \\
\hline CYP2B6 & $* 6$ & $\mathrm{G} / \mathrm{T}$ & Decreased \\
CYP2C9 & $* 2,{ }^{*} 3$ & $* 1 /{ }^{*}$ & Decreased \\
CYP2C19 & $* 2,{ }^{*},{ }^{*} 17$ & $* 1 /{ }^{*}$ & Normal \\
CYP2D6 & $* 2, * 3,{ }^{*} 4,{ }^{*},{ }^{*} 6$, & $* 1 / * 41$ & Normal \\
& $* 7, * 8, * 9, * 10, * 12, * 14,{ }^{*} 17, * 29, * 41, \mathrm{XN}$ & & Normal \\
CYP3A4 & $* 1 \mathrm{~B}$ & $\mathrm{~A} / \mathrm{A}$ & Decreased \\
CYP3A5 & $* 3 \mathrm{C}$ & $\mathrm{G} / \mathrm{G}$ & Decreased \\
\hline
\end{tabular}

Table 3. Treatment plan based on pharmacogenetic analysis

\begin{tabular}{|c|c|c|c|c|c|c|c|c|c|c|c|c|c|c|c|}
\hline \multirow{2}{*}{ Drug } & \multicolumn{15}{|c|}{ Cytochrome P450 } \\
\hline & $1 \mathrm{~A} 1$ & $1 \mathrm{~A} 2$ & 1B1 & $2 \mathrm{~A} 6$ & $2 \mathrm{~B} 6^{*}$ & $2 \mathrm{C} 8$ & $2 \mathrm{C} 9^{*}$ & $2 \mathrm{C} 19$ & 2D6 & $2 \mathrm{E} 1$ & $3 \mathrm{~A} 4$ & $3 \mathrm{~A} 5^{*}$ & $3 \mathrm{~A} 7$ & $4 \mathrm{~B} 1$ & No CYP MDR1* \\
\hline $\begin{array}{l}\text { Aripiprazole } \\
\text { Lithium }\end{array}$ & & & & & & & & & S Ind Inh & & S & S & $S$ & & \\
\hline Bupropion & & S & & & $S \operatorname{Inh}$ & S & S & & $S \operatorname{Inh}$ & S & S & & & & \\
\hline
\end{tabular}

Ind, inductor; Inh, inhibitor; S, substrate; No CYP, no use of P450 pathway.

*Enzymes altered in this patient.

ergy transfer (FRET)-probe assays to define fusion curves.

The pharmacogenetic study showed a possible decrease in the expression of MDR1 transport protein/multidrug resistance protein (heterozygous genotype carrier of allele T of 3435 C > T of MDR1 gene). A genotype associated with low efficiency of CYP2B6 (G/T), CYP2C9 $\left(* 1 /{ }^{*}\right)$ and CYP3A5 $(\mathrm{G} / \mathrm{G})$ was also identified. By contrast, CYP2D6 (alleles $* 1 / * 41$ ), CYP2C19 $\left({ }^{*} 1 / * 1\right.$ ) and CYP3A4 (A/A) were identified, which gives cause to expect an efficient metabolism of the enzymes encoded by these genes.

After several months of near-constant admissions and different drug trials, the lack of clinical response led to a complex polypharmacologic treatment plan (Table 1). Guided by the theoretical interaction assessment and the results yielded by a pharmacogenetic analysis, the treatment regimen was modified:

- Clonazepam, which can interact with carbamazepine and olanzapine via 2B6 (altered in this patient) and $3 \mathrm{~A} 4$, was changed to lorazepam, which is not metabolized via the CYP 450 pathway. This was later discontinued.

- Paliperidone was then suspended due to low tolerability, partly caused by low expression of the efflux transporter MDR1 alteration, which increase bio- availability and brain levels of paliperidona. ${ }^{19)}$

- Olanzapine was also discontinued due to lack of response and side effects, mainly sedation and weight gain. This could be related to MDR1 and 2C9 alteration in this patient, as well as to interaction with carbamazepine and nicotine via $1 \mathrm{~A} 2$.

- Carbamazepine was stopped because of its adverse effect profile (sedation, liver function impairment) and global induction of hepatic metabolism, in this case olanzapine and clonazepam metabolism induction.

- The lithium dosage was maintained at 1,600 mg/day.

- Aripiprazole was started at a medium-dose of 15 $\mathrm{mg} /$ day.

- Afterwards, as the patient started to show signs of a depressive episode, he was started on bupropion, which he took for several months at a dose of 300 $\mathrm{mg} /$ day.

The ultimate goal was to improve the metabolic profile associated to polypharmacy and thereby the efficacy of the treatment, also reducing its side effects, especially weight gain, sedation and apathy.

During the two months following modification of the treatment regimen, a progressive overall clinical improvement became apparent, as well as a reduction of side 
effects.

Five months after starting progressive medication changes based on the pharmacogenetic study, a more simplified treatment (Table 3) was found to be effective in relieving the patient's symptoms: he regained euthymia, recovered his normal functional behaviour and mental organization, and showed more initiative and an improvement in cognitive performance.

Similarly, irritability and insomnia disappeared.

In terms of healthcare resource consumption and overall recovery of functionality, the patient has not required any further hospital admissions; he is living with his parents and has become more involved in both household and social activities.

\section{DISCUSSION}

Antipsychotic treatment efficacy can be influenced by clinical, environmental and social factors, thus explaining the high interindividual variability in drug response and tolerability.

Such heterogeneity in response can also be associated with genetic factors. Consequently, pharmacogenetic studies should be considered when prescribing drugs that are metabolized via cytochrome P450 or use protein transporter such as MDR1. Similarly, the assessment of the pharmacological effect will depend on whether or not the drug is administered as a prodrug. ${ }^{20)}$

Regarding the current case, we would like to highlight three elements: 1) a severe and prolonged manic episode with persistent disabling symptoms that required admission for more than five months, and poor therapeutic response; 2) low tolerance to medication with significant side effects and, consequently, poor adherence to treatment $^{21)}$; and 3) large consumption of healthcare resources, with 89 days of hospitalization over the last five months.

\section{P450 CYP1A2 Induction}

Prior to the pharmacogenetic study, we assessed the pattern of theoretical drug interactions in the patient and found two potential stimulators of metabolism: nicotine and carbamazepine, which have been described as inducers of the activity of these enzymes. Specifically, carbamazepine can induce the expression of CYP1A2, CYP2B6, CYP2C9, CYP2C19, CYP3A4 and CYP3A5.
Tobacco consumption, on the other hand, can induce CYP1A1 and CYP1A2. The inductive effect on CYP1A2 by both carbamazepine and nicotine could accelerate the metabolism and, thus, reduce both the efficacy and adverse effects of psychotropic drugs using CYP1A2 (the main metabolism pathway of olanzapine). ${ }^{22)}$ From this point of view, it is also tempting to interpret high tobacco consumption as an attempt to reduce the powerful drug induced side effects suffered by the patient. The potential consequences of this CYP1A2 induction could be: 1) decreased plasma levels of olanzapine, with a reduction of plasma concentration/dose ratio of up to $36 \%^{23)}$ that could explain therapeutic resistance; and 2) olanzapine metabolism impairment leading to a variation in the 4-N-desmethylolanzapine-olanzapine ratio that could justify the significant olanzapine related side effects observed in this case. It should be noted that the pharmacokinetics of olanzapine is affected by a wide interindividual variability, resulting in a large variation in plasma concentrations of olanzapine for a same dose, so that an individualized dosage is required to avoid both the occurrence of side effects associated with plasma concentrations (obesity, increased intake, sedation), and therapeutic resistance. $^{24)}$

\section{Low Efficiency of CYP2C9, CYP2B6 and CYP3A5}

The pharmacogenetic study revealed that the patient is a carrier of genetic variants that predict low efficiency of CYP2C9, CYP2B6 and CYP3A5. In addition, the inhibitory effect of olanzapine on CYP2C19, CYP2C9 and CYP3A4 could cause phenocopy, which would lead to an accumulation of drug metabolized by these pathways, and thus increase the severity of adverse effects.

\section{MDR1 Genetic Variant}

Another remarkable result yielded by genetic analysis is the detection of a variation of the Efllux transporter MDR1 pump (heterozygotic genotype carrier of the allele of 3435 C > T polymorphism of MDR1 gene) that could predict significant variation in its efficiency. ${ }^{25)}$ The MDR1 gene encodes P-gp, an ATP-dependent membrane transport protein that regulates the bioavailability of multiple drugs. ${ }^{25)}$ The presence of a T allele suggests an impairment of gene-expression that could increase brain levels of drugs that use this carrier pump. It's the case of paliperidona which is substrate of MDR1 and its disposition is in- 
fluenced by the functional status of MDR1. ${ }^{19)}$

In fact, this mutation has been found to be related to the severity of symptomatology, indicated by higher scores on the Positive and Negative Syndrome Scale. ${ }^{26)}$ In general, most antipsychotics act as P-gp inhibitors and can thus influence both plasma and brain drug concentrations. ${ }^{27)}$ The existence of polymorphic variations of P-gp modifies the bioavailability of psychoactive drugs ${ }^{27)}$ and the $3435 \mathrm{C}>\mathrm{T}$ variant has been observed to correlate with raised plasma concentrations of paliperidone. ${ }^{20)}$

Regarding this, olanzapine, and paliperidone to some extent, could compete with other drugs and effectively block MDR1, causing elevated brain concentrations of drugs whose efflux is inhibited. Nevertheless, paliperidone is not metabolized by P450 cytochrome complex. ${ }^{28)}$ However, the inhibitory effect of olanzapine on CYP3A enzyme, as previously mentioned, combined with MDR1 pump alteration, can cause an increase in drug interactions, as proved by in vitro tests. ${ }^{29)}$ This justifies discontinuation of paliperidone in this patient, since, although it does not interfere with CYP1A2, CYP2C9, and CYP3A5 isoenzymes, it is a substrate for the MDR1 pump, which could make it difficult to control their bioavailability.

Accordingly, it seems that patients with this genotype taking olanzapine have greater needs of social and mental health care. ${ }^{30)}$ Furthermore, dose-related concentrations of olanzapine in plasma and cerebrospinal fluid significantly increase, which would also contribute to the side effects observed in this patient. ${ }^{31)}$

\section{CYP3A4 and CYP2D6}

Finally, the pharmacogenetic study indicates that CYP3A4 and CYP2D6 have a pattern of efficient metabolism. CYP2D6 is responsible for the oxidative metabolism of up to $25 \%$ of drugs, such as antidepressants, antipsychotics, opioids, antiarrhythmic agents and tamoxifen in its prodrug form. Its activity is highly variable, being primarily responsible for defining patients as extensive, deficient, intermediate or ultrarapid metabolizers. ${ }^{32)}$ This great variability is explained, at least partly, because it is an enzyme whose genetic locus can have more than 100 variants and sub-variants. ${ }^{33,34)}$ However, in this case it does not seem to play a significant role, as it has little effect on olanzapine plasma concentrations ${ }^{24)}$ and does not participate in the metabolism of paliperidone. Thus, de- spite the great importance of this enzyme in the metabolism of psychotropic drugs, it does not play a role in the clinical situation of this patient.

To summarize, the results of the pharmacogenetic study helped us to redefine a new therapeutic plan in a more rational way.

First, we tried to liberate the most compromised enzymes, namely CYP1A2, CYP2C9, CYP2C19 and CYP3A4, by eliminating olanzapine.

Secondly, although paliperidone has a better side effects profile and does not use the P450 pathway, its toxicity may be explained by the MDR1 alteration, which made discontinuation advisable.

Thirdly, aripiprazole was selected as the best antipsychotic option because of its good metabolic profile, fewer sedative effects and the fact that it is not affected by MDR1 pump dysfunction. ${ }^{35)}$ Thus, the medication plan was simplified and the daily dose reduced (lithium 1,200 $\mathrm{mg} /$ day, and aripiprazole, $15 \mathrm{mg} /$ day).

Finally, the choice of bupropion as an antidepressant was based on its clinical profile, as it has fewer effects on the sexual sphere, can help to reduce nicotine consumption and its interaction potential with antipsychotics is low. ${ }^{22)}$ Bupropion and aripiprazole are both partly metabolized by CYP2D6, which functioned normally in this patient. ${ }^{36)}$ Nonetheless, it is known that when this enzyme is inhibited, bupropion can be metabolized by other routes. $^{37)}$ In addition, bupropion is not affected by CYP2C9 genotypic alterations and can still use CYP2C19 enzyme for its metabolism, so its use in this patient was expected to produce clinical benefits and few tolerance problems. ${ }^{38)}$ Most importantly, bupropion is considered to carry a low risk of inducing mania, especially when it is taken in combination with lithium. ${ }^{39)}$

On the other hand, bupropion could inhibit aripiprazole metabolism, raising the latter's plasma concentrations to up to $45 \%,{ }^{40,41)}$ so it would be prudent to use a low-medium dose of aripiprazole while combined with bupropion.

A last important consideration is that none of the psychotropic drugs used in the final prescription plan is influenced by MDR1 dysfunctionality.

These results highlight the important contribution clinical pharmacogenetic studies make towards predicting responses to psychotropics and understanding individual variation in such clinical response to these drugs. ${ }^{42,43)}$ In 
addition, this case is an example where pharmacogenetic assessment has a significant impact on reducing costs of mental health care. This has also been observed in other studies, in which cost reduction by the use of pharmacogenetic studies can reach up to $28 \%{ }^{44)}$ and, hence, guidelines recommend its use to reduce the high costs associated to mental health care. ${ }^{8,44)}$

In our experience, pharmacogenetic analysis proved a very useful guide in choosing an appropriate treatment plan to manage this complex clinical case. Therefore, its use with patients with a history of poor response and extreme or unusual intolerance to standard drug treatment, once plausible explanations are ruled out, is worth considering. ${ }^{20)}$ Incorporating pharmacogenetic assessment to clinical practice would be useful to optimize pharmacological treatment of mental disorders ${ }^{13)}$ and provide a more personalized therapeutic approach, based on the growing knowledge of the function and effect of genetic polymorphisms on the pharmacokinetics and pharmacodynamics of psychotropic drugs. ${ }^{45)}$ Certainly, other genes can be involved and more studies are needed to assess the implications of different factors in the efficacy, tolerability and interindividual variability of response to psychiatric drugs. ${ }^{46)}$ Pharmacogenetics provides a useful tool for further progress towards offering a more personalized medicine, which is particularly necessary in complex clinical cases like the one reported. ${ }^{47)}$

\section{REFERENCES}

1. Kodesh A, Goldshtein I, Gelkopf M, Goren I, Chodick G, Shalev V. Epidemiology and comorbidity of severe mental illnesses in the community: findings from a computerized mental health registry in a large Israeli health organization. SoC Psychiatry Psychiatr Epidemiol 2012;47:1775-1782.

2. Angst J, Paksarian D, Cui L, Merikangas KR, Hengartner MP, Ajdacic-Gross V, et al. The epidemiology of common mental disorders from age 20 to 50: results from the prospective Zurich cohort study. Epidemiol Psychiatr Sci 2016;25:24-32.

3. Nuss P, de Carvalho W, Blin P, Arnaud R, Filipovics A, Loze $\mathrm{JY}$, et al. [Treatment practices in the management of patients with bipolar disorder in France. The TEMPPO study]. Encephale 2012;38:75-85. French.

4. Costa e Silva JA. Personalized medicine in psychiatry: new technologies and approaches. Metabolism 2013;62 Suppl 1:S40-S44.

5. Samer CF, Lorenzini KI, Rollason V, Daali Y, Desmeules JA. Applications of CYP450 testing in the clinical setting. Mol Diagn Ther 2013;17:165-184.
6. Nivoli AM, Colom F, Pacchiarotti I, Murru A, Scott J, Valentí $\mathrm{M}$, et al. Treatment strategies according to clinical features in a naturalistic cohort study of bipolar patients: a principal component analysis of lifetime pharmacological and biophysic treatment options. Eur Neuropsychopharmacol 2013;23:263275.

7. Bernardo M, Vieta E, Saiz Ruiz J, Rico-Villademoros F, Alamo C, Bobes J; Grupo RECAP. [Recommendations for switching antipsychotics. A position statement of the Spanish Society of Psychiatry and the Spanish Society of Biological Psychiatry]. Rev Psiquiatr Salud Ment 2011;4:150-168. Spanish.

8. U.S. Food and Drug Administration. Table of pharmacogenomic biomarkers in drug labeling [Internet]. Silver Spring, MD: U.S. Food and Drug Administration; 2015. Lcited at 2015 Jul 27]. Available from: http://www.fda.gov/Drugs/ ScienceResearch/ResearchAreas/Pharmacogenetics/ucm083 378.htm.

9. Winner J, Allen JD, Altar CA, Spahic-Mihajlovic A. Psychiatric pharmacogenomics predicts health resource utilization of outpatients with anxiety and depression. Trans/ Psychiatry 2013;3:e242.

10. Stingl JC, Brockmöller J, Viviani R. Genetic variability of drug-metabolizing enzymes: the dual impact on psychiatric therapy and regulation of brain function. Mol Psychiatry 2013;18:273-287.

11. Hiemke C, Pfuhlmann B. Interactions and monitoring of antipsychotic drugs. Handb Exp Pharmacol 2012;(212):241-265.

12. Ravyn D, Ravyn V, Lowney R, Nasrallah HA. CYP450 pharmacogenetic treatment strategies for antipsychotics: a review of the evidence. Schizophr Res 2013;149:1-14.

13. Cacabelos R, Martínez-Bouza R. Genomics and pharmacogenomics of schizophrenia. CNS Neurosci Ther 2011;17:541565.

14. Nakamura A, Mihara K, Nemoto K, Nagai G, Kagawa S, Suzuki T, et al. Lack of correlation between the steady-state plasma concentrations of aripiprazole and haloperidol in Japanese patients with schizophrenia. Ther Drug Monit 2014; 36:815-818.

15. Eiermann B, Engel G, Johansson I, Zanger UM, Bertilsson L. The involvement of CYP1A2 and CYP3A4 in the metabolism of clozapine. Br J Clin Pharmacol 1997;44:439-446.

16. Trenton A, Currier G, Zwemer F. Fatalities associated with therapeutic use and overdose of atypical antipsychotics. CNS Drugs 2003;17:307-324.

17. Zhang JP, Malhotra AK. Pharmacogenetics and antipsychotics: therapeutic efficacy and side effects prediction. Expert Opin Drug Metab Toxicol 2011;7:9-37.

18. Ingelman-Sundberg M, Sim SC, Gomez A, Rodriguez-Antona C. Influence of cytochrome P450 polymorphisms on drug therapies: pharmacogenetic, pharmacoepigenetic and clinical aspects. Pharmacol Ther 2007;116:496-526.

19. Zhu HJ, Wang JS, Markowitz JS, Donovan JL, Gibson BB, DeVane CL. Risperidone and paliperidone inhibit p-glyco- 
protein activity in vitro. Neuropsychopharmacology 2007;32: 757-764.

20. Suzuki Y, Tsuneyama N, Fukui N, Sugai T, Watanabe J, Ono $\mathrm{S}$, et al. Impact of the $A B C B 1$ gene polymorphism on plasma 9-hydroxyrisperidone and active moiety levels in Japanese patients with schizophrenia. J Clin Psychopharmacol 2013; 33:411-414.

21. Perera V, Gross AS, Polasek TM, Qin Y, Rao G, Forrest A, et al. Considering CYP1A2 phenotype and genotype for optimizing the dose of olanzapine in the management of schizophrenia. Expert Opin Drug Metab Toxicol 2013;9:1115-1137.

22. Dervaux A, Laqueille X. [Tobacco and schizophrenia: therapeutic aspects]. Encephale 2007;33:629-632. French.

23. Olesen OV, Linnet K. Olanzapine serum concentrations in psychiatric patients given standard doses: the influence of comedication. Ther Drug Monit 1999;21:87-90.

24. Söderberg MM, Dahl ML. Pharmacogenetics of olanzapine metabolism. Pharmacogenomics 2013;14:1319-1336.

25. Wang D, Johnson AD, Papp AC, Kroetz DL, Sadée W. Multidrug resistance polypeptide 1 (MDR1, ABCB1) variant $3435 C>T$ affects mRNA stability. Pharmacogenet Genomics 2005; 15:693-704.

26. Almoguera B, Riveiro-Alvarez R, Lopez-Castroman J, Dorado P, Vaquero-Lorenzo C, Fernandez-Piqueras J, et al. CYP2D6 poor metabolizer status might be associated with better response to risperidone treatment. Pharmacogenet Genomics 2013;23:627-630.

27. Moons T, de Roo M, Claes S, Dom G. Relationship between P-glycoprotein and second-generation antipsychotics. Pharmacogenomics 2011;12:1193-1211.

28. de Leon J, Wynn G, Sandson NB. The pharmacokinetics of paliperidone versus risperidone. Psychosomatics 2010;51: 80-88.

29. Yasuda K, Lan LB, Sanglard D, Furuya K, Schuetz JD, Schuetz EG. Interaction of cytochrome P450 $3 \mathrm{~A}$ inhibitors with P-glycoprotein. J Pharmacol Exp Ther 2002;303:323-332.

30. Alenius $M$, Wadelius $M$, Dahl ML, Hartvig $P$, Lindström L, Hammarlund-Udenaes M. Gene polymorphism influencing treatment response in psychotic patients in a naturalistic setting. J Psychiatr Res 2008;42:884-893.

31. Skogh E, Sjödin I, Josefsson M, Dahl ML. High correlation between serum and cerebrospinal fluid olanzapine concentrations in patients with schizophrenia or schizoaffective disorder medicating with oral olanzapine as the only antipsychotic drug. J Clin Psychopharmacol 2011;31:4-9.

32. Gaedigk A. Complexities of CYP2D6 gene analysis and interpretation. Int Rev Psychiatry 2013;25:534-553.

33. Mas $S$, Gassò $P$, Alvarez $S$, Parellada E, Bernardo M, Lafuente A. Intuitive pharmacogenetics: spontaneous risperidone dosage is related to CYP2D6, CYP3A5 and ABCB1 genotypes. Pharmacogenomics J 2012;12:255-259.

34. Gaedigk A, Riffel AK, Berrocal BG, Solaesa VG, Dávila I, Isidoro-García M. Characterization of a complex CYP2D6 genotype that caused an AmpliChip CYP450 Test no-call in the clinical setting. Clin Chem Lab Med 2014;52:799-807.

35. Suzuki T, Mihara K, Nakamura A, Kagawa S, Nagai G, Nemoto K, et al. Effects of genetic polymorphisms of CYP2D6, CYP3A5, and ABCB1 on the steady-state plasma concentrations of aripiprazole and its active metabolite, dehydroaripiprazole, in Japanese patients with schizophrenia. Ther Drug Monit 2014;36:651-655.

36. Sager JE, Tripathy S, Price LS, Nath A, Chang J, StephensonFamy A, et al. In vitro to in vivo extrapolation of the complex drug-drug interaction of bupropion and its metabolites with CYP2D6; simultaneous reversible inhibition and CYP2D6 downregulation. Biochem Pharmacol 2017;123:85-96.

37. Benowitz NL, Zhu AZ, Tyndale RF, Dempsey D, Jacob P 3rd. Influence of CYP2B6 genetic variants on plasma and urine concentrations of bupropion and metabolites at steady state. Pharmacogenet Genomics 2013;23:135-141.

38. Zhu AZ, Zhou Q, Cox LS, Ahluwalia JS, Benowitz NL, Tyndale RF. Gene variants in CYP2C19 are associated with altered in vivo bupropion pharmacokinetics but not bupropion-assisted smoking cessation outcomes. Drug Metab Dispos 2014;42:1971-1977.

39. Pacchiarotti I, Bond DJ, Baldessarini RJ, Nolen WA, Grunze $\mathrm{H}$, Licht RW, et al. The International Society for Bipolar Disorders (ISBD) task force report on antidepressant use in bipolar disorders. Am J Psychiatry 2013;170:1249-1262.

40. Castberg I, Spigset O. Effects of comedication on the serum levels of aripiprazole: evidence from a routine therapeutic drug monitoring service. Pharmacopsychiatry 2007:40:107110.

41. Waade RB, Christensen H, Rudberg I, Refsum H, Hermann M. Influence of comedication on serum concentrations of aripiprazole and dehydroaripiprazole. Ther Drug Monit 2009;31: 233-238.

42. Brandl EJ, Chowdhury NI, Tiwari AK, Lett TA, Meltzer HY, Kennedy JL, et al. Genetic variation in CYP $3 A 43$ is associated with response to antipsychotic medication. I Neural Transm (Vienna) 2015;122:29-34.

43. Kim KA, Joo HJ, Lee HM, Park JY. Influence of $A B C B 1$ and CYP3A5 genetic polymorphisms on the pharmacokinetics of quetiapine in healthy volunteers. Pharmacogenet Genomics 2014;24:35-42.

44. Herbild L, Andersen SE, Werge T, Rasmussen HB, Jürgens G. Does pharmacogenetic testing for CYP45O 2D6 and 2C19 among patients with diagnoses within the schizophrenic spectrum reduce treatment costs? Basic Clin Pharmacol Toxicol 2013;113:266-272.

45. Ackenheil M, Weber K. Differing response to antipsychotic therapy in schizophrenia: pharmacogenomic aspects. Dialogues Clin Neurosci 2004;6:71-17.

46. Marazziti D, Baroni S, Picchetti M, Piccinni A, Carlini M, Vatteroni $\mathrm{E}$, et al. Pharmacokinetics and pharmacodynamics of psychotropic drugs: effect of sex. CNS Spectr 2013;18: 
118-127.

47. Wilffert B, Swen J, Mulder H, Touw D, Maitland-Van der Zee $\mathrm{AH}$, Deneer $\mathrm{V}$; KNMP working group Pharmacogenetics.
From evidence based medicine to mechanism based medicine. Reviewing the role of pharmacogenetics. Int J Clin Pharm 2011;33:3-9. 